\title{
Efficient $\omega-k$-algorithm for circular SAR and cylindrical reconstruction areas
}

\author{
A. Dallinger, S. Schelkshorn, and J. Detlefsen \\ Technische Universität München, Lehrstuhl für Hochfrequenztechnik, Fachgebiet Hochfrequente Felder und Schaltungen, \\ D-80333 München, Germany
}

\begin{abstract}
We present a novel reconstruction algorithm of $\omega-k$ type which suits for wideband circular synthetic aperture data taken in stripmap mode. The proposed algorithm allows to reconstruct an image on a cylindrical surface. The range trajectory is approximated by Taylor Series expansion using only the quadratic terms which limits the angular reconstruction range (cross range). In our case this is not a restriction for the application. Wider areas with respect to cross range can be realized by joining several reconstructed images side by side to build a wider image by means of digital spotlighting.
\end{abstract}

\section{Introduction}

Broadband imaging in three dimensions is feasible using the scattered field distributions measured along curved lines. As a special case also circular apertures can provide 3-D spatial resolution (Ishimaru et al., 1998). A circular aperture can be realized by either using an array of sensors or by moving one sensor on a circular trajectory generating a synthetic aperture of circular shape.

In general holographic data can be focused for a known aperture and reconstruction area geometry as long as the sampling constraints are met. A direct inversion scheme of the linearized inverse scattering problem can be realized e. g. by means of correlation or backprojection algorithms. These algorithms are not optimum with respect to computational efficiency but have the advantage to work easily with arbitrary geometries.

Fast and efficient algorithms ( $\omega-k$ type) exist for focusing spotlight circular synthetic aperture data on planes parallel to the circular aperture plane (Soumekh, 1996). In the context of imaging of humans it would be desirable to

Correspondence to: A. Dallinger

(alexander.dallinger@tum.de) reconstruct scattering centers primarily on cylindrical surfaces. Therefore we present an efficient $\omega-k$ stripmap reconstruction algorithm adapted for focusing holographic data, taken by a monostatic circular aperture, onto a cylindrical reconstruction area. This type of imaging is related to linear stripmap slant range imaging (Soumekh, 1999), but the derivation of the spectral support function of the scattering equation relies on an approximation of the range trajectory. This limits the length of the circular aperture and the size of the reconstructed area. In our case this is not a severe restriction because target signatures usually cannot be observed over wider angular regions compared to the limits given by the approximation. If wider angular regions have to imaged so-called digital spotlighting can be used in order to join several small stripmap images side by side. Also reconstruction areas lying within a widespread scattering area can be imaged using this technique.

\section{Derivation of the algorithm}

\subsection{Geometrical definitions}

Figure 1 shows the geometrical setup. A monostatic broadband imaging sensor is moved around the object on a circular path. The angular path variable is $\varphi_{\mathrm{a}}$. For convenience we assume that the range of $\varphi_{\mathrm{a}}$ is symmetric with respect to the $x-z$-plane, i.e. $\varphi_{\mathrm{a}} \in\left[-\varphi_{\mathrm{a}, \max } \ldots \varphi_{\mathrm{a}, \max }\right]$. The path length in angular coordinates is $L_{\varphi_{\mathrm{a}}}=2 \varphi_{\mathrm{a}, \max }$ and the radius $\rho_{\mathrm{a}}$. We also assume that the sensor illuminates the object homogeneously, i.e. the object resides within the [3] dB antenna beamwidth. The scattered field is sampled on the discretized circular path with an angular increment of $\Delta \varphi_{\mathrm{a}}$. The object is reconstructed on a cylindrical surface with radius $\rho_{\mathrm{o}}$ and an angular extent of $L_{\varphi_{\mathrm{o}}}=2 \varphi_{\mathrm{o} \text {, max }}$ with $\varphi_{\mathrm{o}} \in\left[-\varphi_{\mathrm{o}, \max } \ldots \varphi_{\mathrm{o}, \max }\right]$. 


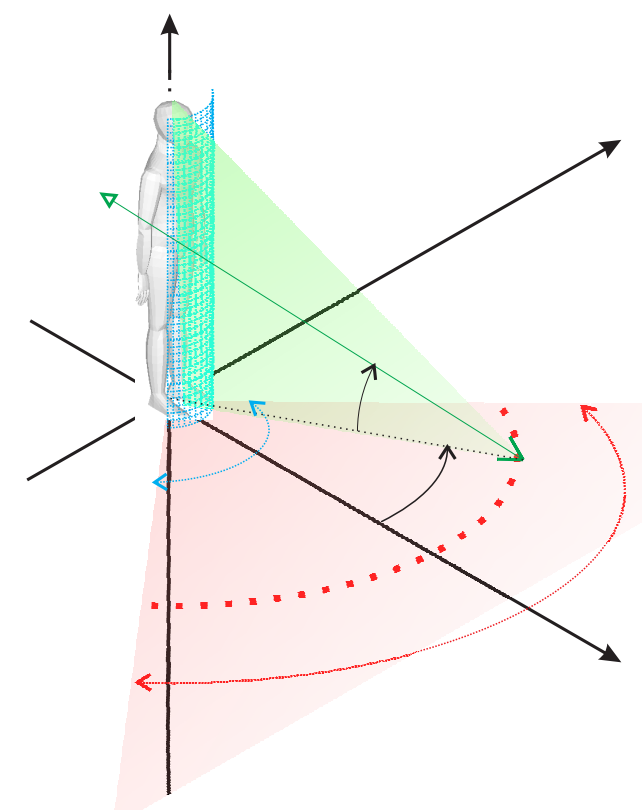

Fig. 1. SAR imaging geometry: The imaging sensor is moved on a circular path around the person. It is assumed that the person is illuminated homogeneously. The imaging area is located on a cylindrical surface.

Both the aperture and the reconstruction area are described in polar coordinates, that is for the aperture $\boldsymbol{r}_{\mathrm{a}}=\left(\rho_{\mathrm{a}} \cos \varphi_{\mathrm{a}}, \rho_{\mathrm{a}} \sin \varphi_{\mathrm{a}}, z_{\mathrm{a}}\right)^{\top}$ and for the reconstruction area $\boldsymbol{r}_{\mathrm{O}}=\left(\rho_{\mathrm{o}} \cos \varphi_{\mathrm{o}}, \rho_{\mathrm{o}} \sin \varphi_{\mathrm{o}}, z_{\mathrm{o}}\right)^{\top}$.

\subsection{Linearized inverse scattering}

The first order Born approximation (Morse and Feshbach, 1953 ) is used in order to linearize the inverse scattering problem. The description of the object may be further simplified by assuming distributed point sources which do not interact with each other in an electromagnetic sense. Thus the scattering of the incident wave reduces to a single bounce, line of sight (direct path between the transmitter/receiver and the point scatterer) process, which is linear. Hence contributions from each point scatterer superimpose coherently. The scattered field (monostatic case, i.e. the incident wavevector $\boldsymbol{k}_{\mathrm{i}}$ is equal to the scattered wavevector $-\boldsymbol{k}_{\mathrm{s}}$ ) is found to be

$u_{\mathrm{s}}\left(\boldsymbol{r}_{\mathrm{a}}, k\right)=\int o\left(\boldsymbol{r}_{\mathrm{o}}\right) \exp \left[-j 2 k r\left(\boldsymbol{r}_{\mathrm{a}} \mid \boldsymbol{r}_{\mathrm{o}}\right)\right] \mathrm{d} \boldsymbol{V}_{\mathrm{o}}$.

The object itself is described by an object function $o\left(\boldsymbol{r}_{\mathrm{o}}\right)$ which is assumed to be a superposition of distributed point sources, i.e. $o\left(\boldsymbol{r}_{\mathrm{o}}\right)=\sum_{n=1}^{N} \delta_{n}\left(\boldsymbol{r}_{\mathrm{o}, n}\right) . k$ stands for the wavevector of the propagation medium. For the monostatic case we have $k=|\boldsymbol{k}|=\left|\boldsymbol{k}_{\mathrm{i}}\right|=\left|-\boldsymbol{k}_{\mathrm{s}}\right|=2 \pi / \lambda=2 \pi f / c_{0}$. The support of $k$ is given by the bandwidth $B$ of the system, i.e. $k \in\left[2 \pi f_{\min } / c_{0} \ldots 2 \pi f_{\max } / c_{0}\right]$.
The distance from the aperture to a point of the reconstruction area can be determined by

$r\left(\boldsymbol{r}_{\mathrm{a}} \mid \boldsymbol{r}_{\mathrm{o}}\right)$

$\sqrt{\rho_{\mathrm{a}}^{2}+\rho_{\mathrm{o}}^{2}-2 \rho_{\mathrm{a}} \rho_{\mathrm{o}} \cos \left(\varphi_{\mathrm{o}}-\varphi_{\mathrm{a}}\right)+\left(z_{\mathrm{o}}-z_{\mathrm{a}}\right)^{2}}$.

\subsection{Angular spectral domain of a point scatterer}

The inversion of Eq. 1 leads to the unknown object function $o\left(\boldsymbol{r}_{\mathrm{o}}\right)$. For an $\omega-k$ type inversion one has to find the spectral support function of a point scatterer with respect to the path variable. Therefore we approximate Eq. 2 by using a Taylor series expansion of the cosine term (i.e. $\left.\cos x=1-\frac{x^{2}}{2 !}+\ldots\right)$. By neglecting higher order terms the distance becomes

$r\left(\boldsymbol{r}_{\mathrm{a}} \mid \boldsymbol{r}_{\mathrm{o}}\right)=\sqrt{\alpha+\beta\left(\varphi_{\mathrm{o}}-\varphi_{\mathrm{a}}\right)^{2}}$

with $\alpha=\left(\rho_{\mathrm{o}}-\rho_{\mathrm{a}}\right)^{2}+\left(z_{\mathrm{o}}-z_{\mathrm{a}}\right)^{2}$ and $\beta=\rho_{\mathrm{a}} \rho_{\mathrm{o}}$. The scattered signal measured at the receiver is

$u_{s}\left(\boldsymbol{r}_{\mathrm{a}}, k\right)=u_{S}\left(\varphi_{\mathrm{a}}, k\right)=e^{-j 2 k \sqrt{\alpha+\beta\left(\varphi_{\mathrm{o}}-\varphi_{\mathrm{a}}\right)^{2}}}$.

$\boldsymbol{r}_{\mathrm{a}}$ depends in this case only on the cross range angle $\varphi_{\mathrm{a}}\left(\rho_{\mathrm{a}}\right.$ and $z_{\mathrm{a}}$ are constant). By taking the Fourier transform of both sides of Eq. 4 with respect to the variable $\varphi_{\mathrm{a}}$ the angular spectral function is obtained to

$$
\begin{aligned}
u_{S}\left(k_{\varphi_{\mathrm{a}}}, k\right) & =\mathcal{F}_{\varphi_{\mathrm{a}}}\left\{u_{S}\left(\varphi_{\mathrm{a}}, k\right)\right\} \\
& =\int_{-\pi}^{\pi} e^{-j 2 k r} e^{-j k_{\varphi_{\mathrm{a}}} \varphi_{\mathrm{a}}} d \varphi_{\mathrm{a}} .
\end{aligned}
$$

The integral can be evaluated by the method of stationary phase. The general result is given by

$$
\int_{-\infty}^{\infty} g(\chi) e^{j \phi(\chi)} d \chi=\sqrt{\frac{j 2 \pi}{\phi^{\prime \prime}\left(\chi^{*}\right)}} g\left(\chi^{*}\right) e^{j \phi\left(\chi^{*}\right)} .
$$

$\chi^{*} \in \chi$ is called the stationary point and is determined by the solution of $\phi^{\prime}\left(\chi^{*}\right)=0$. The stationary point $\varphi_{\mathrm{a}}^{*}$ of Eq. 5 can be determined to be

$\varphi_{\mathrm{a}}^{*}=\varphi_{\mathrm{o}}-\frac{\sqrt{\alpha} k_{\varphi_{\mathrm{a}}} / \beta}{\sqrt{4 k^{2}-k_{\varphi_{\mathrm{a}}}^{2} / \beta}}$.

The angular spectrum follows to

$u_{S}\left(k_{\varphi_{\mathrm{a}}}, k\right)=e^{-j \sqrt{4 k^{2}-k_{\varphi_{\mathrm{a}}}^{2} / \beta} \sqrt{\alpha}-j k_{\varphi_{\mathrm{a}}} \varphi_{\mathrm{o}}}$

neglecting all changes of amplitude terms.

\subsection{Inversion scheme}

The term $\sqrt{\alpha}$ in Eq. 8 is the distance of closest approach from the sensor to a point scatterer $\left(\varphi_{\mathrm{a}}=\varphi_{\mathrm{o}}\right)$. It is used as the range axis $r_{\tau}$ (in terms of propagation delay $\tau=2 r / c_{0}$ ). 
For the reconstructed image the range and cross range variables, $r_{\tau}$ and $\varphi$, respectively, are used (whereas $\varphi=\varphi_{\mathrm{a}}$ ). The spacial spectrum of point scatterer $n$ at position $\left(r_{\tau, n}, \varphi_{\mathrm{o}, n}\right)$ can be described by

$\mathcal{F}_{\varphi, r_{\tau}}\left\{\delta\left(\varphi-\varphi_{\mathrm{o}, n}, r_{\tau}-r_{\tau, n}\right)\right\}=e^{-j k_{r_{\tau}} r_{\tau, n}-j k_{\varphi} \varphi_{\mathrm{o}, n}}$.

Comparing Eqs. 8 and 9 and taking into account Eq. 1 the inversion scheme results in

$o\left(r_{\tau}, \varphi\right)=\mathcal{F}_{k_{\varphi}, k_{r_{\tau}}}^{-1}\left\{\right.$ Stolt Mapping $\left.\left[\mathcal{F}_{\varphi_{\mathrm{a}}}\left\{u_{S}\left(\varphi_{\mathrm{a}}, k\right)\right\}\right]\right\}$.

The so called Stolt Mapping is a variable transformation which is described by

$$
\begin{aligned}
k_{r_{\tau}} & =\sqrt{4 k^{2}-k_{\varphi_{\mathrm{a}}}{ }^{2} / \beta} \\
k_{\varphi} & =k_{\varphi_{\mathrm{a}}} .
\end{aligned}
$$

After having performed the Stolt Mapping an interpolation of the data, which is distributed on a non-linear grid $\left(k_{r_{\tau}}, k_{\varphi}\right)$ is necessary. Methods like the Nonuniform Fourier transform (NUFFT), (Fessler and Sutton, 2003) or Gridding (Jackson et al., 1991; Wajer et al., 2000) can be applied. We use NUFFT because precomputed interpolation matrices can be used which makes the computational process very fast.

The inversion scheme is straight forward and has close similarities to the case when a linear monostatic aperture is used (Soumekh, 1999; Carrara et al., 1995).

\subsection{Angular spectral support}

The support of the angular spectrum can be estimated by calculating the angular spectral variable $k_{\varphi_{\mathrm{a}}}$ which is

$$
\begin{aligned}
k_{\varphi_{\mathrm{a}}} & =\frac{d L\left\{u_{s}\left(\varphi_{\mathrm{a}}, k\right)\right\}}{d \varphi_{a}} \\
& =\frac{2 k \rho_{\mathrm{a}} \rho_{\mathrm{o}} \sin \left(\varphi_{\mathrm{o}}-\varphi_{\mathrm{a}}\right)}{\sqrt{\rho_{\mathrm{a}}^{2}+\rho_{\mathrm{o}}^{2}-2 \rho_{\mathrm{a}} \rho_{\mathrm{o}} \cos \left(\varphi_{\mathrm{o}}-\varphi_{\mathrm{a}}\right)+\left(z_{\mathrm{o}}-z_{\mathrm{a}}\right)^{2}}} .
\end{aligned}
$$

The angular spectral support band is determined by the angular extent of the reconstruction area and the length of the aperture. Assuming that $L_{\varphi_{\mathrm{a}}}=L_{\varphi_{\mathrm{o}}}$ the boundaries of the support band can be estimated from Eq. 12 which results in

$$
\left|k_{\varphi_{\mathrm{a}}}\right| \leq \frac{2 k_{\max } \rho_{\mathrm{a}} \rho_{\mathrm{o}} \sin \left(L_{\varphi_{\mathrm{a}}}\right)}{\sqrt{\rho_{\mathrm{a}}^{2}+\rho_{\mathrm{o}}^{2}-2 \rho_{\mathrm{a}} \rho_{\mathrm{o}} \cos \left(L_{\varphi_{\mathrm{a}}}\right)+\left(z_{\mathrm{o}}-z_{\mathrm{a}}\right)^{2}}} .
$$

Due to the strong chirp character of the received signal the angular spectrum of a point scatterer is a bandpass function. The boundaries are calculated by using Eq. 12. Assuming a point scatterer at position $\varphi_{0}=0$ its angular spectral support is given by

$$
\begin{aligned}
& \left|k_{\varphi_{\mathrm{a}}, n}\right| \\
& \leq \frac{2 k_{\max } \rho_{\mathrm{a}} \rho_{\mathrm{o}} \sin \left(L_{\varphi_{\mathrm{a}}} / 2\right)}{\sqrt{\rho_{\mathrm{a}}^{2}+\rho_{\mathrm{o}}^{2}-2 \rho_{\mathrm{a}} \rho_{\mathrm{o}} \cos \left(L_{\varphi_{\mathrm{a}}} / 2\right)+\left(z_{\mathrm{o}}-z_{\mathrm{a}}\right)^{2}}},
\end{aligned}
$$

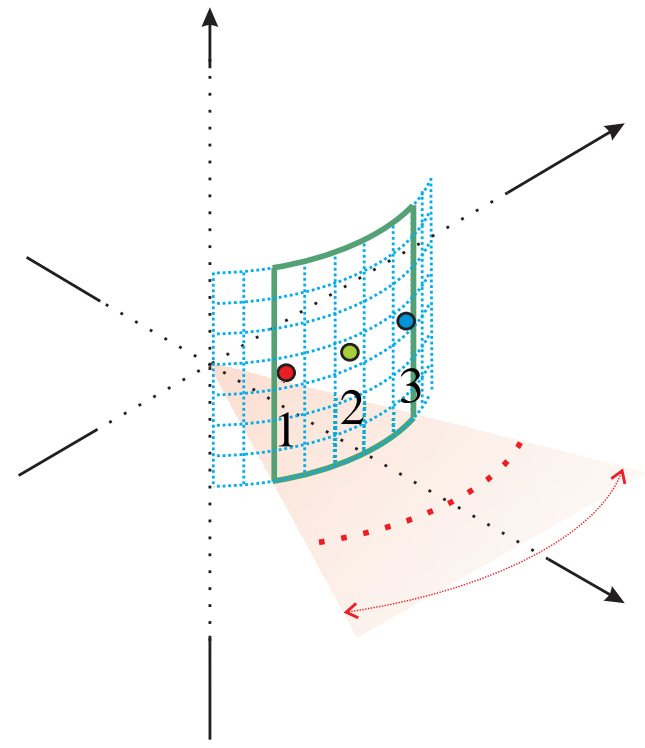

(a)
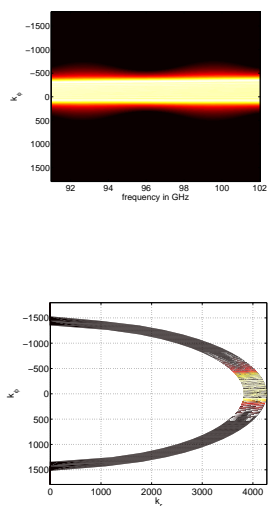

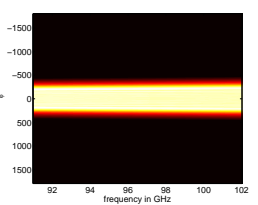

(b)

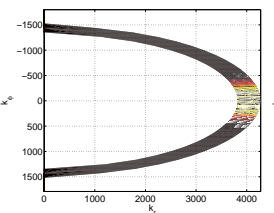

(c)

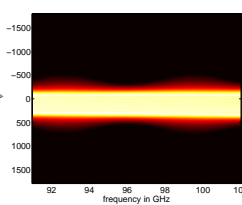

trequency in G $^{9}$

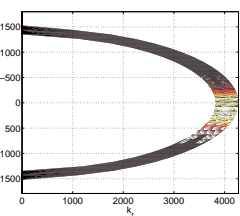

Fig. 2. The spectrum of point scatterers with different angular positions $\left(L_{\varphi}=[40]^{\circ}, \Delta \varphi=[0.1]^{\circ}, \rho_{\mathrm{a}} / \rho_{\mathrm{O}}=3,\left|z_{\mathrm{a}}-z_{\mathrm{o}}\right|=[0.5] \mathrm{m}\right.$, $B=[11] G H z)$

(a) setup of three point scatterers (numbered from 1 to 3 ) placed on a cylindrical surface (blue grid) within the reconstruction area (green cylindrical boundary), (b) angular spectrum vs. frequency $u_{\mathrm{S}}\left(k_{\varphi_{\mathrm{a}}}, k\right)$, (c) angular spectrum vs. the spectral range domain $u_{\mathrm{S}}\left(k_{\varphi_{\mathrm{a}}}, k_{r_{\tau}}\right)$

The left column belongs to point scatterer $1\left(\varphi_{\mathrm{O}}=[-10]^{\circ}\right)$, the middle column to scatterer $2\left(\varphi_{\mathrm{o}}=[0]^{\circ}\right)$ and the right column to scatterer $3\left(\varphi_{0}=[10]^{\circ}\right)$.

which can be used to calculate the angular resolution (see Sect. 2.6.2).

The center of the angular spectrum is depending on the actual position of the point scatterer with respect to its angular position. Figure 2 shows the spectrum $u_{\mathrm{s}}\left(k_{\varphi_{\mathrm{a}}}, k\right)$ of three point scatterers placed on a cylindrical surface but with different angular positions. 


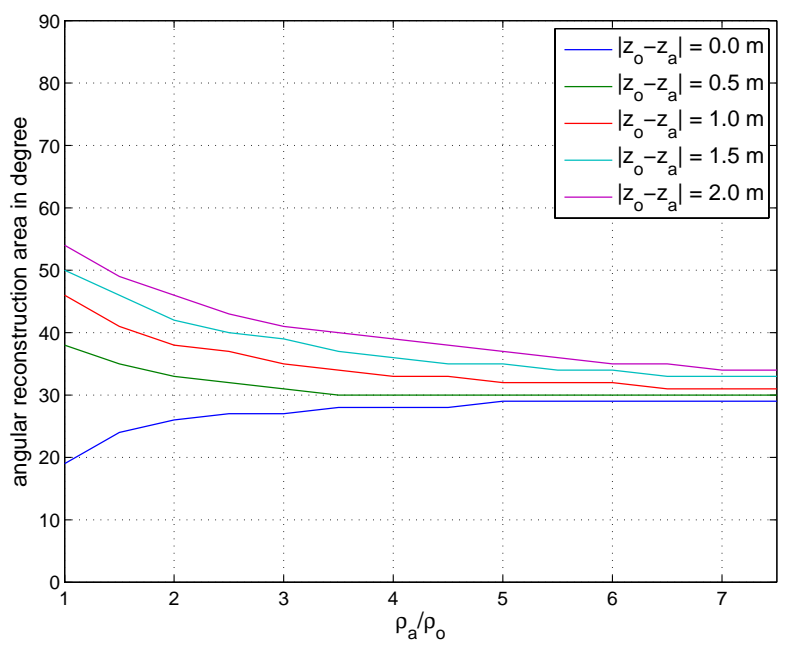

Fig. 3. Estimation of the valid angular reconstruction area respective the normalized radius $\rho_{\mathrm{a}} / \rho_{\mathrm{O}}$. The tolerated phase error is $[10]^{\circ}$.

\subsection{Resolution}

\subsubsection{Range resolution}

The range resolution $\Delta r_{\tau}$ is given by the bandwidth $B$ of the radar system and is calculated by $\Delta r_{\tau}=c_{0} / 2 B$, with $c_{0}$ the free space propagation velocity. The resolution in z-direction follows to be $\Delta z=\Delta r_{\tau} / \sin \theta, \theta$ is the inclination angle of the antenna (see Fig. 1).

\subsubsection{Cross range resolution}

The cross range resolution can be estimated from the extension of the angular spectral support band of a single point scatterer Eq. 14, i.e. $\Delta \varphi=\pi /\left|k_{\varphi_{\mathrm{a}}, n}\right|$.

\section{Validity of the algorithm respective the cross range approximation}

Due to the approximation of the cosine term in Eq. 3 the expression for the angular spectrum is only valid within a limited angular range. Figure 3 shows the valid region for a cylindrical reconstruction area versus the normalized distance $\rho_{\mathrm{a}} / \rho_{\mathrm{o}}$ assuming the phase difference of the approximated signal Eq. 3 with respect to the ideal signal Eq. 1 to be smaller than ten degrees.

Since the approximation gets worse with increasing angular domain the quality of the image will gradually degrade as the distance to the center of the image plane is increased. This effect is illustrated in Fig. 4 where an array of point scatterers is reconstructed.

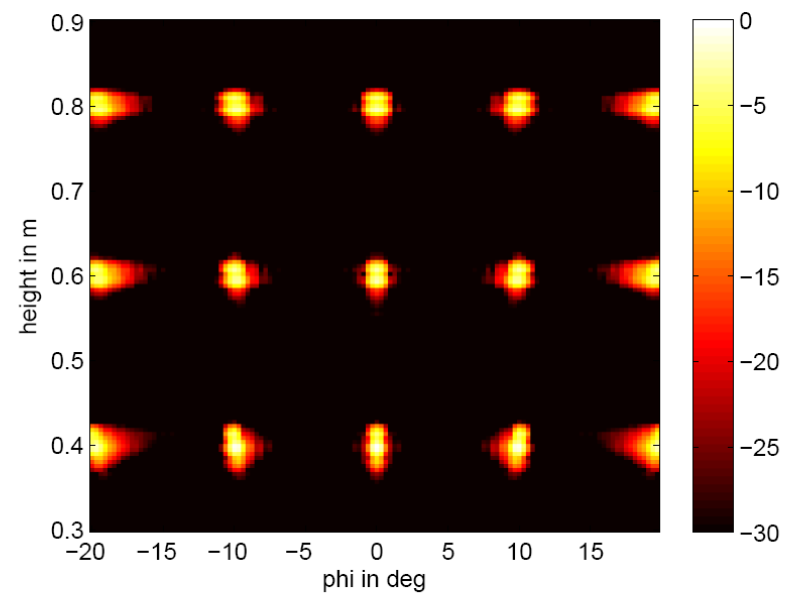

Fig. 4. Reconstructed image of point scatterers in log. scale $\left(L_{\varphi}=[40]^{\circ}, \Delta \varphi=[0.1]^{\circ}, \rho_{\mathrm{a}} / \rho_{\mathrm{o}}=3, B=[11] \mathrm{GHz}\right)$ : the angular resolution gets worse with increasing angular offset to the center of the image (the range resolution enhances with increasing range which is directly related to the height $z_{\mathrm{o}}$ of the point scatterer placed on a cylindrical surface)

\section{Digital spotlighting}

\subsection{Circular convolution characteristics}

Scatterers placed outside the unambiguous range are reconstructed as ghost targets. This effect can be attributed to the circular convolution characteristics of the discrete Fourier transform.

The same occurs in cross range direction. Since the size of the synthetic aperture is $L_{\varphi_{\mathrm{a}}}$ the sample spacing in the angular spectral domain is $\Delta k_{\varphi_{\mathrm{a}}}=2 \pi / L_{\varphi_{\mathrm{a}}}$. The cross range mapping in the spatial frequency domain is defined by $k_{\varphi}=k_{\varphi_{\mathrm{a}}}$, i.e. the resultant samples of the angular spectrum of the object function $o\left(k_{\varphi}\right)$ are also separated by $\Delta k_{\varphi_{\mathrm{a}}}=2 \pi / L_{\varphi_{\mathrm{a}}}$. The angular length of the illuminated target area may be $L_{\varphi_{0}}{ }^{\prime}$ (including also scatterers lying outside the reconstruction area). To avoid aliasing in the $\varphi$ domain, the sample spacing in the $k_{\varphi}$ domain should satisfy the Nyquist rate $\Delta k_{\varphi} \leq 2 \pi / L_{\varphi_{0}}{ }^{\prime}$. If the size of the target area is greater than the synthetic aperture length, i.e. $L_{\varphi_{0}}{ }^{\prime}>L_{\varphi_{\mathrm{a}}}$ than $o\left(k_{\varphi}\right)$ will be aliased. Scatterers placed outside the angular reconstruction boundary as illustrated in Fig. 3 show up as ghost targets, see Fig. 3.

\subsection{Zero-padding in angular direction}

By increasing the length of the aperture $L_{\varphi_{\mathrm{a}}}$ by means of zero-padding $u_{\mathrm{s}}\left(\varphi_{\mathrm{a}}, f\right)$ with respect to the angular variable $\varphi_{\mathrm{a}}$ an effective aperture with length $L_{\varphi_{\mathrm{a}}{ }^{\prime}}=L_{\varphi_{0}}{ }^{\prime}$ is obtained and $\Delta k_{\varphi_{\mathrm{a}}}=\Delta k_{\varphi}$ which means that $o\left(k_{\varphi}\right)$ will not be aliased. This operation has no influence on the resolution of the image but increases the computational load which can be reduced with the following method. 


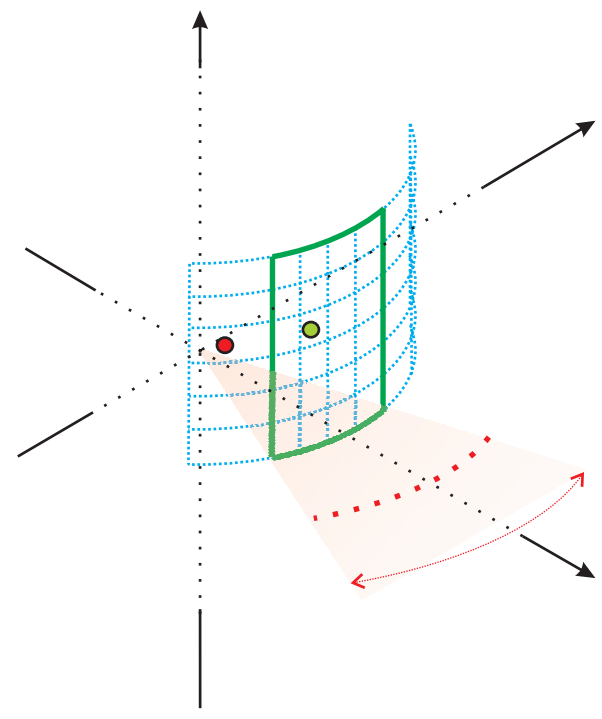

(a)

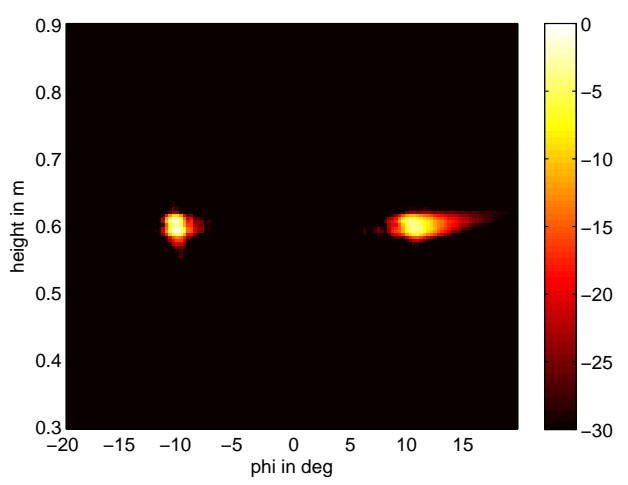

(b)

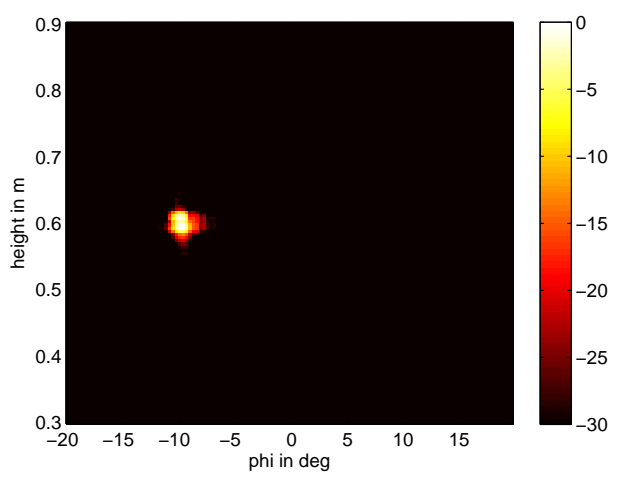

(c)

Fig. 5. Digital Spotlighting $\left(L_{\varphi}=[40]^{\circ}, \Delta \varphi=0.1^{\circ}, \rho_{\mathrm{a}} / \rho_{\mathrm{o}}=3\right.$, $B=[11] G H z)$ : (a) two point scatterers placed on a cylindrical surface: scatterer 1 lies in the reconstruction area at $\left(\varphi_{\mathrm{o}, 1}=[-10]^{\circ}\right.$, $\left.z_{\mathrm{o}, 1}=[0.6] \mathrm{m}\right)$, scatterer 2 at $\left(\varphi_{\mathrm{o}, 2}=[-30]^{\circ}, z_{\mathrm{o}, 2}=[0.6] \mathrm{m}\right)$, (b) scatterer 2 shows up as a ghost target in the reconstructed image, (c) zero padding in the angular direction increases the unambiguous angular region, the ghost target is gone

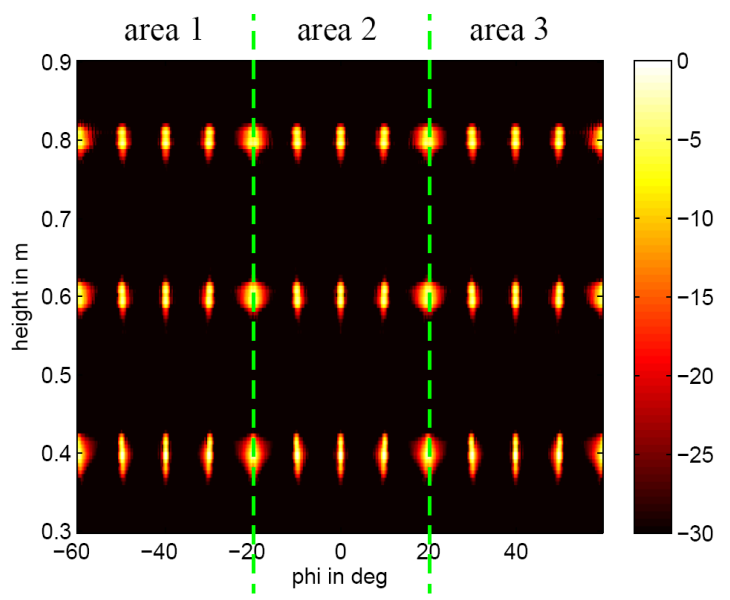

Fig. 6. Three reconstruction areas joined side by side to obtain a wide cross range image $\left(L_{\varphi}=3 \times[40]^{\circ}\right.$, $\left.\Delta \varphi=[0.1]^{\circ}, \rho_{\mathrm{a}} / \rho_{\mathrm{o}}=3, B=[11] G H z\right)$. The SAR data $u_{\mathrm{S}}\left(\varphi_{\mathrm{a}}, f\right)$ is divided in 3 parts of equal length and zero-padded by a factor of two. Additionally a rectangular bandpass filters out the appropriate spectral support region

\subsection{Filtering in angular spectral domain}

To image a reconstruction area embedded inside a wider scattering area (digital spotlighting) one method is to apply a bandpass filter in the angular spectral domain. According to Sect. 2.5 the boundaries of the angular spectral support of the reconstruction area can be calculated by Eq. 12 . The spectral support of a point scatterer is a bandpass function. The spectral overlap of the reconstruction area and the spectrum of the illuminated scatterer lying outside is determined by the angular position of these scatterer. A bandpass operation which filters out the spectral support of the reconstruction area will attenuate the ghost target but also slightly degrade the image quality.

In combination with the method introduced in Sect. 4.2 the filter-width can be wider and an optimum solution respective the digital spotlighting task can be found which does not lead to an image degradation.

In reality the beamwidth of the antenna pattern also helps to filter out the area of interest.

\subsection{Subaperture imaging and image joining}

The angular approximation used to derive the angular spectral support function limits the imaging area in angular direction. Using subaperture digital spotlighting techniques smaller angular segments can be processed and the results can be joined side by side in order to obtain a wider imaging area with approx. a constant resolution all over the total image.

Figure 6 shows an image of a wide angular reconstruction area. Three areas have been processed by subaperture digital spotlighting and joined side by side. 
Table 1. Parameter settings of data acquisition using a homodyne FMCW radar

\begin{tabular}{lll}
\hline frequency range & $f_{\min } \ldots f_{\max }$ & {$[91] \mathrm{GHz} \ldots[102] \mathrm{GHz}$} \\
bandwidth & $B$ & {$[11] \mathrm{GHz}$} \\
range resolution & $\Delta r$ & {$[1.36] \mathrm{mm}$} \\
sweeptime & $T$ & {$[2] \mathrm{ms}$} \\
TX power & $P_{\text {out }}$ & {$[20] \mathrm{dBm}$} \\
RX sampling rate & $f_{\mathrm{s}}$ & {$[500] \mathrm{kHz}$} \\
\hline aperture radius & $\rho_{\mathrm{a}}$ & {$[0.60] \mathrm{m}$} \\
aperture height & $z_{\mathrm{a}}$ & {$[0] \mathrm{m}$} \\
aperture length & $L_{\varphi_{\mathrm{a}}}$ & {$[-90]^{\circ} \ldots[90]^{\circ}$} \\
angular increment & $\Delta \varphi_{\mathrm{a}}$ & {$[0.1]^{\circ}$} \\
angular image length & $L_{\varphi}$ & {$[40]^{\circ}$} \\
\hline radius cyl. imaging area & $\rho_{\mathrm{O}}$ & {$[21.5] \mathrm{cm}$} \\
resolution z direction & $\Delta z$ & {$[15.7] \mathrm{mm}$} \\
resolution cross range & $\Delta \varphi$ & $\approx[2.5] \mathrm{mm}$
\end{tabular}

\section{Imaging results}

In order to validate the derived algorithm and the related techniques an ultrawideband FMCW homodyne radar has been used for data acquisition. The parameter settings are summarized in Table 1.

For validation an artificial object consisting of several small metallic spheres (radius [2]mm) as shown in Fig. 7 has been imaged. The point scatterers have been placed on a PVC cylinder with radius $\rho_{0}=[21.5] \mathrm{cm}$. The reconstruction area has been set to $\varphi_{o}=[-100]^{\circ} \ldots[100]^{\circ}$ and $z_{\mathrm{o}}=[0] \mathrm{m} \ldots[0.6] \mathrm{m}$. The same object also has been imaged with a backprojection algorithm in order to have a reference to compare with.

\section{Conclusions}

We have developed a $\omega-k$ algorithm adapted to circular apertures and cylindrical reconstruction areas. Within certain angular limitations an approximation to the exact spectral support function has been derived which is suitable for broadband imaging applications. We have validated the algorithms both with synthetic and measured real world data obtained with a ultrawideband FMCW radar. No major degradation of the image quality is observed when compared to a reference backprojection algorithm. However the computational efficiency is several orders better and the image shown in Fig. $7 \mathrm{~b}(640 \times 128$ pixels) is computed in about $5 \mathrm{~s}$ (on a $\mathrm{P} 4,1.5 \mathrm{GHz})$.

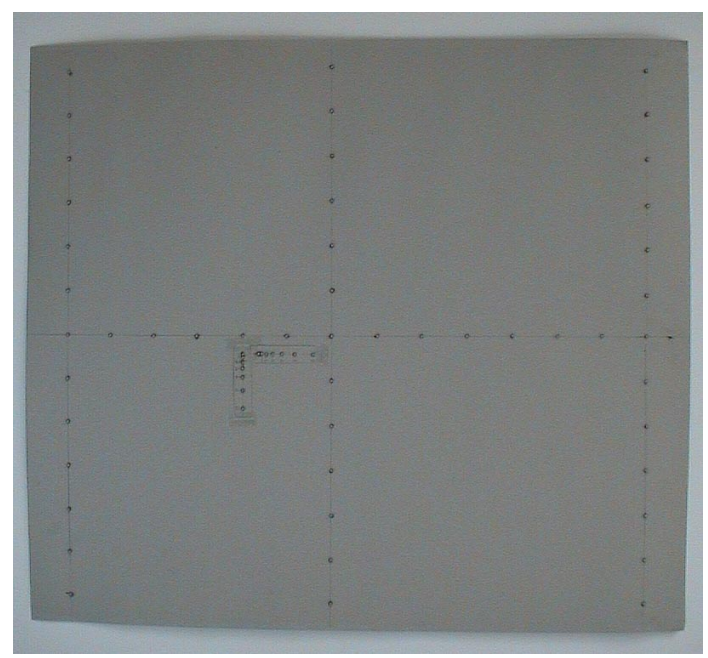

(a) Photo of the object

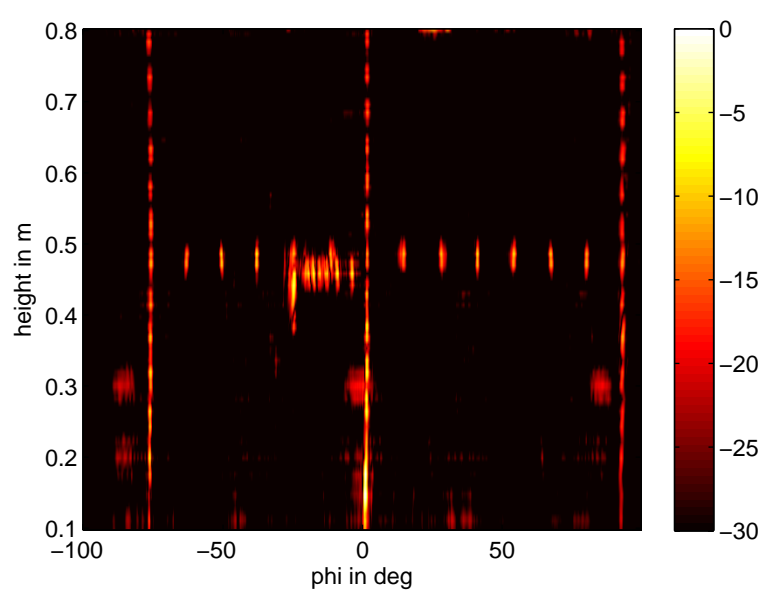

(b) $\omega-k$ : reconstructed image consisting of 5 joined angular areas

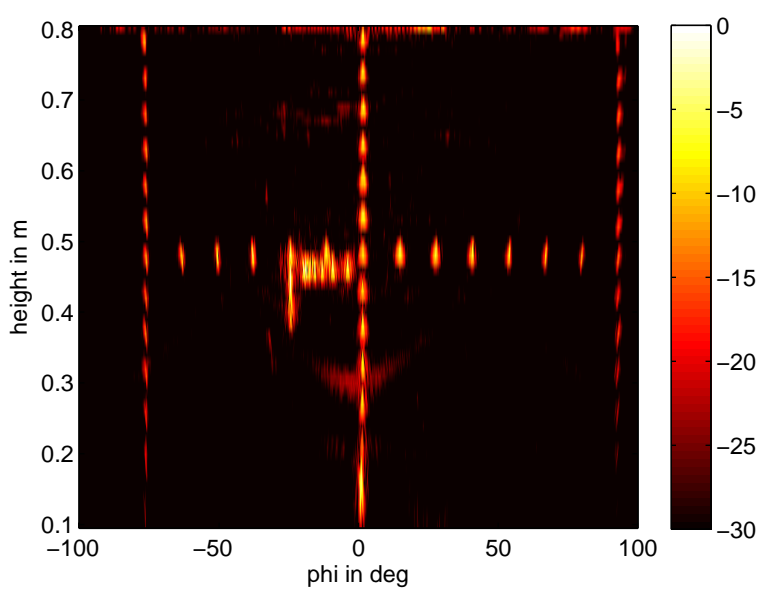

(c) backprojection: reconstructed image

Fig. 7. Small metallic spheres with a radius of [2]mm and a spacing of [5]cm. This structure has been placed on PVC cylinder with radius $\rho_{\mathrm{a}}=[22.5] \mathrm{cm}$ 


\section{References}

Carrara, W. G., Goodman, R. S., and Majewski, R. M.: Spotlight Synthetic Aperture Radar Signal Processing Algorithms, Artech House, 1995.

Fessler, J. A. and Sutton, B. P.: Nonuniform fast Fourier transforms using min-max interpolation, IEEE Transactions on Signal Processing, 51, 560-574, 2003.

Ishimaru, A., Chan, T.-K., and Kuga, Y.: An Imaging Technique Using Confocal Circular Synthetic Aperture Radar, IEEE Transactions on Geoscience and Remote Sensing, 36, 1524-1530, 1998.

Jackson, J. I., Meyer, C. H., Nishimura, D. G., and Macovski, A.: Selection of a convolution function for Fourier inversion using gridding [computerised tomography application], Medical Imaging, IEEE Transactions on, 10, 473-478, 1991.
Morse, P. M. and Feshbach, H.: Methods of Theoretical Physics, vol. Part II, McGraw-Hill Book Company, Inc., 1953.

Soumekh, M.: Reconnaissance with Slant Plane Circular SAR Imaging, IEEE Transactions on Image Processing, 5, 12521265, 1996.

Soumekh, M.: Synthetic Aperture Radar Signal Processing with Matlab Algorithms, John Wiley and Sons, Inc., 1999.

Wajer, F., Lethmate, R., van Osch, J., Graveron-Demilly, D., Fuderer, M., and van Ormondt, D.: Interpolation from Arbitrary to Cartesian Sample Positions: Gridding, in: ProRISC/IEEE workshop, 571-577, 2000. 